\title{
EDITORIAL SPECIAL ISSUE OF SELECTED ARTICLES FROM IRC-SEMS 2016
}

\author{
Xiaoyan Zhu ${ }^{1}$ Qingpei Hu${ }^{2}$ King Pui Louis Liu ${ }^{3}$ Kwai Sang Chin ${ }^{3}$ \\ ${ }^{I}$ University of Chinese Academy of Sciences, Beijing, 100049, China \\ xzhu5@ucas.ac.cn $(\square)$ \\ ${ }^{2}$ Academy of Mathematics and Systems Science, Chinese Academy of Sciences, Beijing, 100190, China \\ qingpeihu@amss.ac.cn \\ ${ }^{3}$ City University of Hong Kong, Hong Kong, China \\ mekpliu@cityu.edu.hk,mekschin@cityu.edu.hk
}

This special issue consists of selected revised and extended versions of papers presented at the International Research Conference on Systems Engineering and Management Science, IRC-SEMS 2016, held in Beijing, China.

The IRC-SEMS annual conference series began in 2013. Since the beginning, it aims to provide a research platform for academics and researchers to share and exchange experience and research findings in various disciplines of systems engineering and management science. The themes include artificial intelligence and expert systems, computer integrated manufacturing systems, decision making, information systems, enterprise resource planning, financial engineering, fuzzy logics, game theory, healthcare systems and management, human factors engineering and ergonomics, industrial statistics, innovation Systems, knowledge and information management, logistics and transportation management, project management, quality systems and reliability engineering, supply chain management and other related topics.
In reflection to the variety of subjects of interest to the community participating at the Conference and in terms of its quality, we have selected potential candidates of the papers for extension and submission to this Journal. After the peer-review process for the selected papers among over 80 papers in total, six were retained.

Xiao, Wang, and Chin (2017) examine the effects of government subsidy as a means to promote remanufacturing activity, particularly considering three subsidy options: subsidy to remanufacturer, subsidy to consumers, and subsidy shared by remanufacturer and consumers. In their paper, a single-period model is established for a system comprised of an original manufacturer who produces only new products and a remanufacturer who collects used products from consumers and produces remanufactured products. It is found that the introduction of government subsidy on remanufacturer or consumers always increases remanufacturing activity and that subsidy to remanufacturer is the best subsidy option.

Chen and Tang (2017) investigate societal 
risk classification using the data source from Tianya Forum. In their paper, four types of representations: string representation, term-frequency representation, TF-IDF representation, and the distributed representation of BBS posts are used. Based on these representations, four k-Nearest Neighbor $(\mathrm{kNN})$ classifiers are developed and compared using edit distance or cosine similarity as distance metric. It is found that $\mathrm{kNN}$ based on the distributed representation generated by Paragraph Vector is effective for societal risk classification and that the performance of societal risk classification can further be improved by taking weighted ensemble of the four kNN classifiers.

Bakker and Tsui (2017) investigate the patient scheduling issue in their paper of "Dynamic resource allocation for efficient patient scheduling: a data-driven approach", with a study conducted for the General Surgery department of a medium-sized hospital. A novel minimal-variation approach, utilizing both discrete-event simulation and empirical data, is proposed for automatic specialist resource calendar optimization. Application study shows the dynamic scheduling approach improves the hospital service quality in terms of Service Level and Wait Time.

Zen, Trimartanti, Abidin, and Abadi (2017) propose a fuzzy approach for "Determining hydrocarbon prospective zone using the combination of qualitative analysis and fuzzy logic method". In addition to the qualitative analysis approach commonly used by geologists, fuzzy logic is applied through fuzzification, inference system, and defuzzification, which improves the accuracy of the analysis.

In Ma, Zhao, and Zhou (2017)'s paper of
"Predicting status of Chinese listed companies based on features selected by penalized regression", the status prediction issue is formulated as a classification problem of dada mining for high dimensional data. Specifically, a logistic regression model is proposed to select interested factors out of more than 150 financial factors. Better prediction accuracy is observed with the analysis results.

In the paper of "Outpatient capacity allocation considering adding capacity to match high patient demand" by Jiang, Tang, and Yan, a study is conducted for the case of where the patients demand is quite higher than the supply. A novel adding capacity policy is proposed to mitigate the mismatch between supply and demand, with a capacity allocation model formulated for both possible no-show routine patients and all show-up same-day patients. Numerical analysis shows better performance of the proposed policy.

We would like to thank all the members of the IRC-SEMS Program Committee, the authors, the external reviewers who provided valuable comments, and the editor of Journal of Systems Engineering and Systems Information, Prof. Jian Chen, who provided great support and assistance in the management of the special issue.

Xiaoyan Zhu is Chair Professor in School of Economics and Management at University of Chinese Academy of Sciences, Beijing since 2014. She received a B.S. degree from Tsinghua University, Beijing, in 2000, and M.S. and Ph.D. degrees in industrial engineering from Texas A\&M University, College Station, USA, in 2002 and 2005, respectively. From 2005 to 2007, she worked as a research associate at the University 
of Tennessee, Knoxville. Since 2008, she worked as Assistant Professor in Industrial and Systems Engineering Department at University of Tennessee, Knoxville, USA. Her research interests are in system reliability optimization, operations research, and supply chain and inventory management. She coauthored book "Importance Measures in Reliability, Risk, and Optimization: Principles and Applications" and published about 30 journal papers in the top journals. She won 2012 IIE Best Paper Prize in Quality Control \& Reliability Engineering. She is a member of Thousand Youth Talents Program of China.

Qingpei Hu received the B.S and M.S. degrees from the Department of Automatic Control, Beijing University of Aeronautics and Astronautics, Beijing, China, in 1999 and 2003, respectively, and the Ph.D. degree in the Industrial and Systems Engineering Department, National University of Singapore, Singapore, in 2007. He is now an Associate Professor in the Academy of Mathematics and Systems Science, Chinese Academy of Sciences, Beijing, China. His current research interests include reliability statistics, systems reliability, and software reliability.

King Pui Louis Liu is currently an instructor at the Department of Systems Engineering and Engineering Management, City University of Hong Kong. He received his $\mathrm{PhD}$ from the same department in 2002. He received his MPhil degree from the University of Hong Kong in 1995, and his Bachelor degree from Portsmouth University, UK in 1991. From 2014 onwards, he has been one of the program co-chairs of the
International Research Conference on Systems Engineering and Engineering Science. Also, he has been a committee member of the International Conference on the Interface between Statistics and Engineering starting from 2014. His research interests are very broad, and they are Service Robotics, Non-destructive Testing, Magnetorheological fluid (MR fluid) application, Giant Magnetostrictive Material (GMM) Application, Simulation Study on System Operation, and Health Care Operation. He has conducted some applied research on remote monitoring and quality assurance of air purifier device installed for patient at home.

Kwai Sang Chin is currently the Associate Head of Department of Systems Engineering and Engineering Management and the Associate Director of Centre for Systems Informatics Engineering at City University of Hong Kong. $\mathrm{He}$ is also serving Chutian Chair Professor in the Wuhan University of Technology, PRC. His teaching specialization and research interests are quality and innovation management, and new product development strategies and process. Dr. Chin graduated from Industrial Engineering of University of Hong Kong. Before joining the City University in 1991, He had 10-year experience in the manufacturing industry, holding senior positions in industrial engineering, product development, and quality assurance and management. Dr. Chin is a Chartered Engineer in UK and Registered Professional Engineer in Hong Kong. He is an Academician of the International Academy of Quality, Fellow of American Society for Quality (ASQ), USA, Fellow of Hong Kong Society for Quality (HKSQ), and senior members of several 
international engineering institutions. Dr. Chin was the Chairman of HKSQ in 1997-2000 and the ASQ Country Representative of Hong Kong 2000-2013, and the founding member of ANQ (Asian Network for Quality) since 2003. He has obtained nearly US\$1.35 million research funding from various sources as Principal Investigator and has published over 180 international refereed journal papers. Due to his strong industrial background, Dr. Chin is maintaining a good link with the industries. 